\title{
أثر التجارة الاككترونية علي عدد المستخدمين في الدول الأفريقية
}

\section{أحمد فلاح صالح مهاوش سلمان}

\author{
طالب دكتوراة بمعهد البحوث والدراسات الافريقية \\ جامعة أسوان
}

تحمه عبدالحفيظ عحم السمان

أستاذ الاقتصاد الزراعي - عميد كلية الزراعة

$$
\text { جامعة سوهاج }
$$

\section{حسن موسي رضوان}

مدرس الاقتصاد الزراعي بالمعهذ العالي للتعاون والارشاد الزراعي بأسيوط 


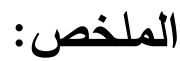

ظهرت التجارة الإلكترونيـة منذ ثلاثة عقود مضت ثم تطور مفهومها خـلال

الربـع الأخيـر مـن القـرن الماضـي بتطـور الأجهزة الإلكترونيـة حيث تخـدم مصـالح

المستهلك، وهنالك الكثير ممن يختلط عليهم معنى التجارة الإلكترونية، حيث يضنون

أنها التجارة في الأجهزة الإككترونية وتوابعها، لكن ليس هذا هو المقصود من التجارة

الإلكترونيـة بـلـ هـي تجـارة كتلـك التجـارة المعروفـة ، أي المعـاملات التـي تـتم بـين

المتعاملين التجاريين، لكن الفرق هو أننا في التجارة الإكترونية نقوم باستخدام أجهزة

ووسائل الإلكترونية مثل الأنترنت ، الفاكس والتلكس.

وقد أشـارت التقارير الأخيره الي أن هنالك مـالا يقل عن اب مليون متسوق

يستخدم شبكه الانترنت في القاره الافريقيه وذلك حتي عام 1 ـ ب في حين ان تعد تلك

النسبه مقارنها بدول العالم فهي تمثل أقل من ب\% من الاجمالي العالمي لمستخدمي

الانترنت.

فهناك تفاوت واضـح في انتشار الانترنت عبر العالم، فنجد قاره أفريقيا تحتل

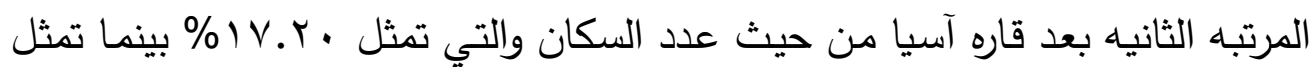
الاخيره $100 \%$ وذلك من اجمالي قارات العالم، كما يتضح ايضاً أن قاره افريقيا تحتل المرتبـه الثالثه من حيث عدد المستعملين او المستخدمين للانترنت بعد قارتي آسيا وأوروبـا، بينمـا تحتل القـاره الافريقيـه المرتبه الآخيـره مـن حيث النسبه المئويـه لعدد 
المستخدمين للانترنت مقارنها باجمالي عدد سكان نفس القاره حيث تمثل ادني نسبه

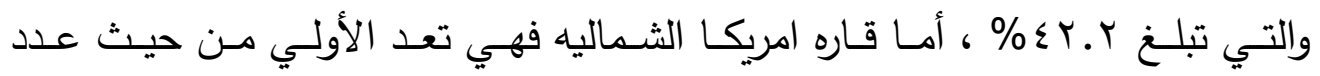
المستعملين للانترنت والتي تمثل r. .9\% ، ويتوسط القارتي الخمس قارات العـالم الاخر وهم أوروبا، أمريكا اللاتينيه، الثرق الأوسط، استراليا، آسيا ، وتقدر النسبه

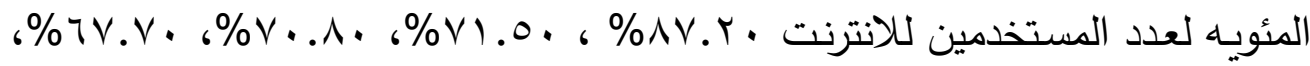

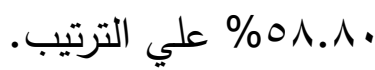
وتظهر الأرقام لدول القاره الافريقيه ان دولة سانت هيلانة تمثل اقل عدد سكان في دول القاره الافريقية حيت تمثل حوالي VV • Y نسمه مـ اجمـالي عدد السكان تقديريا عام • r.r. والتي ليس بها اي مستخدم لثبكه الانترنت علي الاطلاق عام

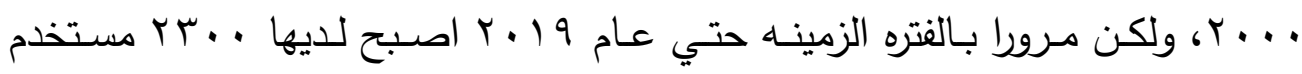
اللثبكه الالكترونية والذي يمثل حوالي rr,A0\% من اجمالي عدد سكانها، ففي عام ل . . . . كانت هذه الدول مايوت، سانت هلانة، جنوب السودان ، الصحراء الغربيه لا يوجد بها اي مستخدم لثبكه الانترنت، وكانت دولة الصومال أقل عددا لمستخدمي للانترنت وكان بها .r مستخدم فقط لشبكه الانترنت بينما اصبح 1,705,300

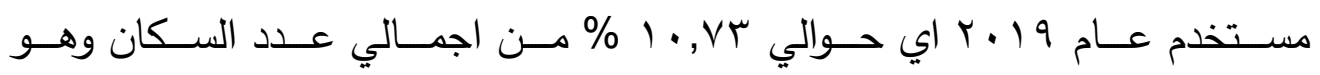
15,893,222 نسمه، اما جنوب أفريقيا فكان يمثل اكبر عدد لمستخدمي الانترت في ذات العام ... ب لدول القاره وهو 2,400,000 شخص وهو علي العكس تماما لدولة 
نيجيريا والتي يبلغ سكانها تقديريا لعام •r.r.r حوالي 206,139,589 نسمه والذي يمثل اكبر عدد للسكان في دول القارة الافريقيه أي حوالي 17 , آ\% من اجمالي عدد السكان ويبلغ عدد مستخدميها للانترنت 126,078,999 شخص بها ، وعلي الرغم من انها الدوله الوحيده الاعلي سكانا في دول القاره الافريقيه الا ان دوله كينيا والتي بها 53,771,296 شخص من اجمالي عدد سكانها حتي عام • r.r. لكن يبلغ عدد مستخدميها للانترنت حوالي 46,870,422 شـخص وهو مـا يمثل عNv, IV مـن اجمالي عدد السكان وهي النسبه الأعلي لمستغلي شبكه الانترنت وهو مايعني أن الغالبيه العظمي من الافراد القانطين بدولة كينيا يستخدمون الانترنت امـا الصحراء الغربيه فكانت اقل نسبه لمستخدمي الانترنت وهي 4.69\% وذلك من اجمالي سكانها التقديري لعام •r.r.r والذي يبلغ 597,339 شخص. ومن هذا المنطلق يتبين مجملاً أن نسبه عدد المستخدمين لشبكه الانترنت للقاره الاقريقيه هي 0. 11\% فقط مقارنه بقارات العالم، وهي نسبه ضئيله جدا تثير الي عدم الاستفاده الكامله من شبكه الانترنت والحصول علي مزاياها لافراد تلك الدول، ومن ثم وجب التعرف علي العقبات التي تواجه دول هذه القاره لنمو التجارة الالكترونية. مما تبين ان هناك العديد من العقبات التي تحول دون نمو التجارة الإكترونية وتقدمها في أفريقيا ومنها ضعف تواصل المواطنين مع شبكات الإنترنت، ضعف البنية 


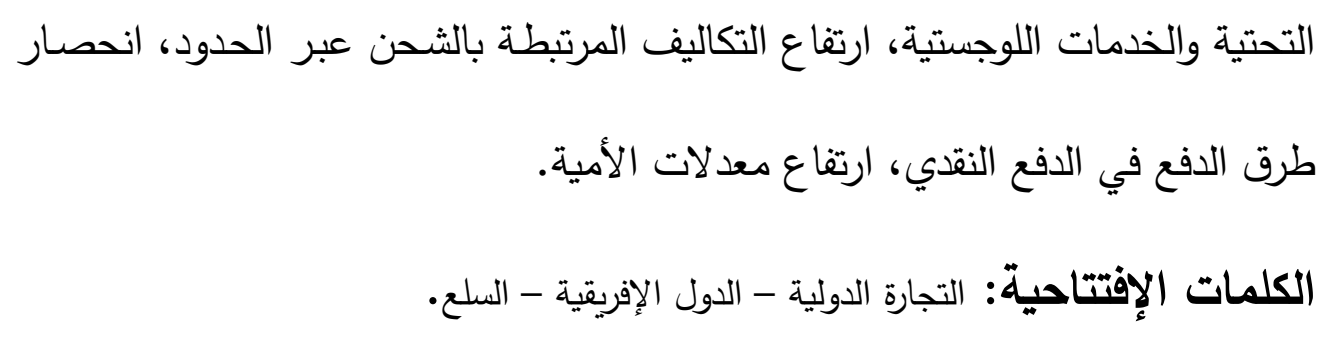

\section{Abstract:}

\section{An effect of E-commerce on the number of users in African countries}

E-commerce appeared three decades ago, then its concept developed during the last quarter of the last century with the development of electronic devices as they serve the interests of the consumer, and there are many who confuse them with the meaning of e-commerce, as they think that it is commerce in electronic devices and their accessories, but this is not what is meant by e-commerce Rather, it is a trade like that known trade, that is, the transactions that take place between commercial dealers, but the difference is that in e-commerce we use electronic devices and means such as the Internet, fax and telex.

Recent reports have indicated that there are at least 21 million shoppers using the Internet in the African continent until 2018, while that percentage is compared to other countries in the world, as it represents less than $2 \%$ of the global total of Internet users. 
There is a clear disparity in the spread of the Internet across the world, so we find Africa occupies the second place after Asia in terms of population, which represents $17.20 \%$ while the latter represents $55.1 \%$ of the total world continents, and it is also evident that the continent of Africa ranks third in terms of the number of users Or Internet users after Asia and Europe, while the African continent ranks last in terms of the percentage of Internet users compared to the total population of the same continent, where it represents the lowest percentage, which is $42.2 \%$, while North America is the first in terms of the number of Internet users, which represents $90.3 \%$, and the middle of the five continents of the other world are Europe, Latin America, the Middle East, Australia, Asia, and the percentage of Internet users is estimated at $87.20 \%, 71.50 \%, 70.80 \%, 67.70 \%, 58.80 \%$, respectively.

The figures for the countries of the African continent show that the state of Saint Helena represents the lowest number of inhabitants in the countries of the African continent, as it represents about 6,077 people of the total population estimated in 2020, which does not have any user of the Internet at all in the year 2000, but through the period until 2019 has become 2300 users of the electronic network, which represents about $37.85 \%$ of its total 
population, in 2000 these countries were Mayotte, Saint Helena, Southern Sudan, Western Sahara, and there was no user of the Internet, and the State of Somalia was the smallest number of Internet users and it was 200 A user only has the Internet, while it became 1,705,300 users in 2019, which is about $10.73 \%$ of the total population, which is $15,893,222$ people. As for South Africa, it was the largest number of Internet users in the same year 2000 for the countries of the continent, which is $2,400,000$ people, which is quite unlike the State of Nigeria, which is Its estimated population for the year 2020 is about $206,139,589$ people, which represents the largest population in the countries of the African continent, or about $61.16 \%$ of the total population, and the number of its Internet users is $126,078,999$ people, although it is $A$, the only country with the highest population in the countries of the African continent, but the country of Kenya, which has $53,771,296$ people of the total population until 2020 , but the number of its users of the Internet is about $46,870,422$ people, which represents $87.1 \%$ of the total population, which is the highest percentage of Internet users This means that the vast majority of people residing in Kenya use the Internet. As for the Western Sahara, the lowest percentage of internet users 
was $4.69 \%$, out of its estimated total population for the year 2020 , which is 597,339 people.

From this point of view it becomes clear that the percentage of the number of users of the Internet on the African continent is only $11.5 \%$ compared to the continents of the world, which is a very small percentage indicating the lack of full use of the Internet and obtaining its advantages for the individuals of those countries, and then it is necessary to identify the obstacles facing these countries Continent to grow electronic commerce.

It turned out that there are many obstacles that prevent the growth and progress of e-commerce in Africa, including poor citizens 'communication with Internet networks, poor infrastructure and logistics, high costs associated with cross-border shipping, payment methods being limited to cash payments, and high illiteracy rates.

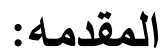

ظهرت التجارة الإلكترونية منذ ثلاثة عقود مضت ثم تطور مفهومها خلال الربـع الأخيـر مـن القـرن الماضـي بتطور الأجهـزة الإلكترونيـة، حيث تخدم مصـالح

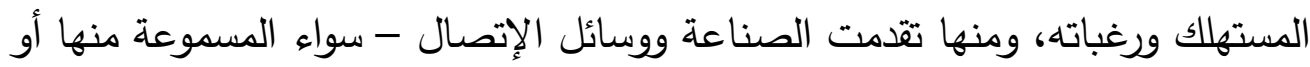
المرئية - مما جعل العالم مجموعة من الدول والثعوب القرببة رغم البعد المكاني بينها، و يتسنى لها أن تطلع على كل المنتجات والإختراعات الحديثة في كل دول العالم عن طريق وسائل الإعلام من خلال الإعلانات التي تقدمها. 
ولقد أدت التطورات السـريعة الحاصـلة في مجـال التجـارة الإكترونيـة ظهور مفاهيم جديدة وتعريفات معينة، يكمن الإختلاف بينها في الزاويـة المنظور منها. وهناك الكثير ممن يختلط عليهم معنى التجارة الإكترونيـة، حيث يظنون أنها التجارة في الأجهزة الإلكترونية وتوابعها، لكن ليس هذا هو المقصود من التجارة الإكترونية بل هي

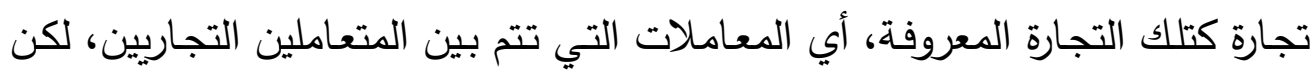

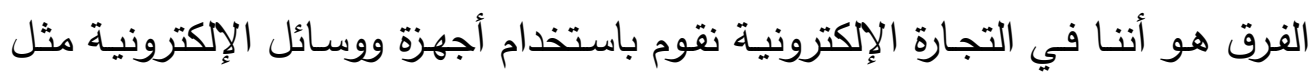
الأنترنت ، الفاكس والتلكس. وقد كثف مسح حديث عن أن غالبية مستخدمي الإنترنت في منطقة الشرق الأوسط وشمال إفريقيا يستخدمون الإنترنت في البحث عن المنتجات، ولكن انتشـار

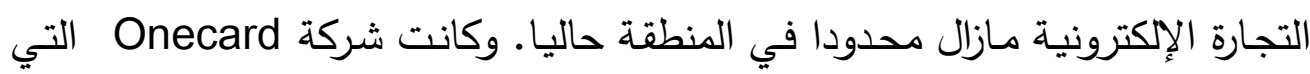
تقدم خدمات الافع عبر الإنترنت، قد استطلعت ألف شخص من مستخدمي الإنترنت

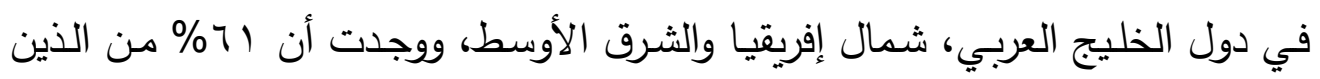
شملهم المسح بحثا عن معلومات تجارية باستخدام الإنترنت، في الوقت الذي قام فيه r \% \% منهم بشراء منتجات عن طريق الإنترنت.

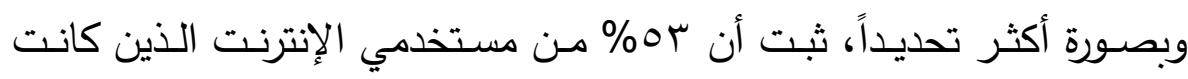
أعمارهم لا تقل عن هr عاما، ونسبة تصل إلى به \% من الذين تتراوح أعمارهم بين

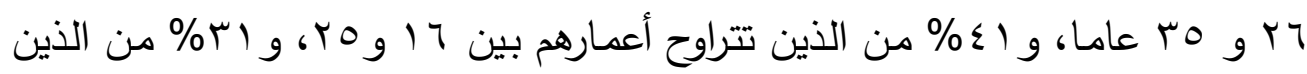
تقل أعمارهم عن 17 عاما كانوا قد انخرطوا في التجارة الإلكترونية. وإجمالا، فقد وجد لـداند المسح بأن ما يقرب من ثلث مستخدمي الإنترنت كانوا قد شاركوا في مثل هذا النشاط،

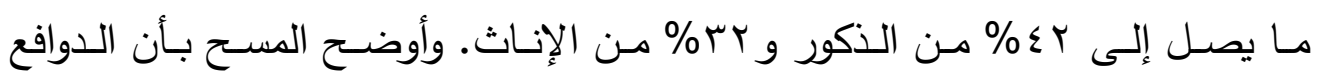
الرئيسية التي تقود الناس للشراء عبر الإنترنت تجيء على النحو التالي: العروض

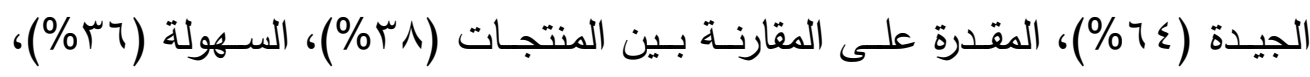
امكانية مراجعة تعليقات الزبائن الموجودة في الموقع حول المنتجات المختلفة (ع ب\%ة)،

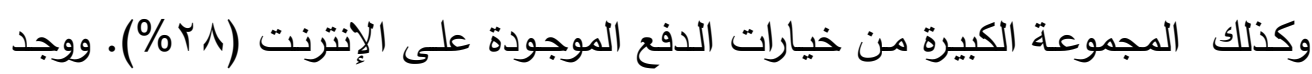
المسح بأن اء٪ من المستطلعين حتى الآن قاموا بشراء العاب أو عملات افتراضية 


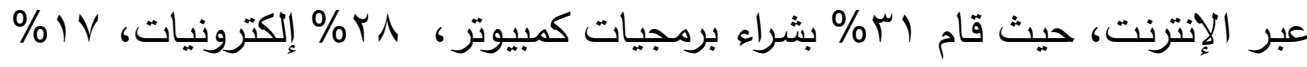

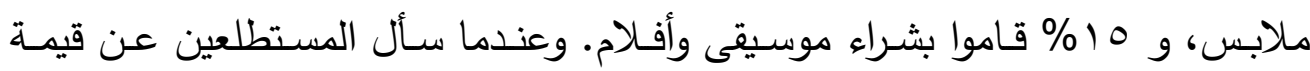

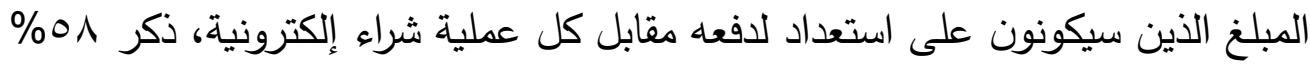
منهم بأنهم على استعداد لدفع ما يصل إلى . . 1 دولار في كل عملية، وهم - غالبا -

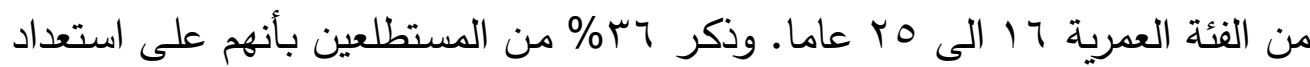
لدفع ما بين .ا إلى ألف دولار في كل عملية شراء إلكترونية ولاسيما في حال توافر

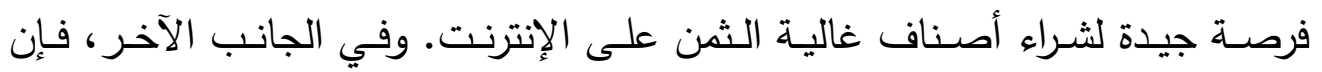

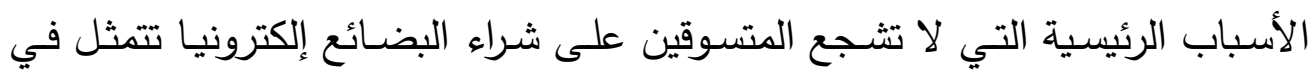
المخاوف من سرقة تفاصيل بطاقاتهم الائتمانية والاحتيال (107\%)، القلق من عملية

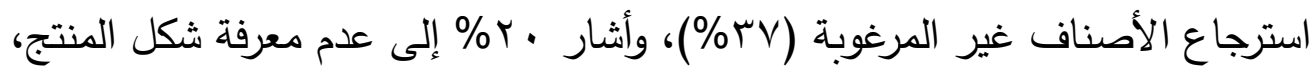
و ذكر 7 ا \% بأنهم لا يمتلكون خبرة حقيقية في الثراء عبر الإنترنت، بينما أقر 9 9 من المستطلعين بحاجتهم إلى نصيحة رجال المبيعات.

\section{مشكلة البحث: - n}

في خضــم التطـورات الهائلــهـ التـي يشـهـها العـالم الآن بـرزت تكنولوجيـا

المعلومات والاتصالات، وتزايد دور التجارة الالكترونية والذي شهد تطورا ملحوظا في السنوات الآخيره من القرن الواحد والعشرون والذي تجلي بدوره الحيوي والفعـال في تقريب المسافات بين الدول والاقراد عبر شبكه الانترنت لما لـه من مزايا كمساهمته في النـاتج المحلي الإجمالي ورفع معدل النمو وزيـاده حجم التبـادل التجاري سواء علي المستوي المحلي اوالاقليمي. مما قد يساهم بشكل اساسي في تحقيق النمو الشامل في مختلـف الأنشـطه والمجـالات ولاسـيما المجـال التجـاري، بالاضـافه الـي تلبيـه كافـه 
الإحتياجات والمتطلبات لدي جميع المستخدمين. إلا أن التقارير الأخيره أشارت الي أن هنآك مالا يقل عن اب مليون متسوق يستخدم شبكه الانترنت في القاره الافريقيه وذلك حتي عام 11 ب ب في حين أن تعد تلك النسبه مقارنه بدول العالم فهي تمثل أقل من r\% من الإجمالي العالمي لمستخدمي الانترنت. لذا كان من الضروري تسليط الضوء علي واقع عدد الدستخدمين لثبكه الانترنت في التجارة الآكترونية وأثرها علي دول القاره الافريقيه وذلك حتي ديسمبر عام ... . و ديسمبر عام 9 ـ. . .

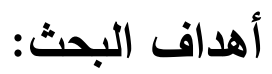

1. تسليط الضوء علي التعرف بالتجارة الالكترونية، خصائصها، وأهميتها.

r. دراسة الأهميه النسبيه لعدد السكان ومستخدمي الإنترنت لقاره افريقيا مقارنهـ

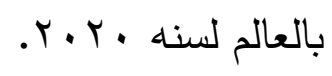

r. التعرف علي الوضح الراهن لعدد السكان ومستخدمي الإنترنت في دول القارة

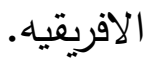

؛. التعرف علي العقبات التي تواجه التجارة الالكترونية في القاره الافريقيه.

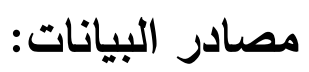
فقد اعتمدت دراسة البحث علي البيانات الصادره من الهيئات الدولية كالأمم المتحده والبنك الدولي وبعض الدوريات والمراجع العربية والاجنبيه السابقة بالاضافه بالاستعانه ببعض المواقع الالكترونية. 


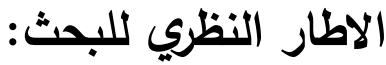

\section{اولاً: ماهيه التجارة الاكترونية:}

تعرف "التجارة الإلكترونية Electronic Commerce بأنها أي شكل من

أشكال التبادل التجاري أو الإداري أو تبادل المعلومات التي تجري باستخدام تقنيات المعلومـات والاتصـالات. كمـا تعرف بأنها تتفيذ مختلف أنشطة الأعمال باستخدام الوسائل المعلوماتية المؤلفة من حواسيب تربطها شبكة حاسوبية لتبادل المعلومات. ويربط بعضهم تعريف التجارة الإلكترونية بشبكة الإنترنت فتعرف بأنها تنفيذ وإدارة الأنثطة التجاريـة المتعلقة بالسلع والخدمات عبر شبكة الإنترنت. أمـا وفقا لمنظمـة التجارة العالمية، "التجارة الالكترونية يشير إلى توزيع الإنتاج، تسويق أو بيع أو تسليم السلع والخدمات بطريقة إلكترونية ".

فإن مفهوم التجارة الإلكترونيـة - commerce التجارية على الثبكات الإككترونية، بما في ذلك تشجيع المبيعات، على الانترنت من المنتجات والخدمات، وخذمة العملاء، وما إلى ذلك. ويشمل ذلك كل من العلاقات بين الثركات بين حكومات ومؤسسات الأعمال والتجارة بين الثركات والمستهلكين. أي المعاملات التجارية تجري كليا أو جزئيا عن طريق الوسائل الإكترونية. كما أن هناك من يـرى أن " التجـارة الإككترونيـة مفهوم عـام يغطي أي نـوع مـن التعامل التجـاري 
المتحقق إلكترونية بإستخدام شبكات الإتصال السلكية منها و اللاسلكية. وتحصل هذه الإتصـالات مـا بين الثركات نفسها أو بينها وبين عمـلاء أو بينها و بين المنظـات

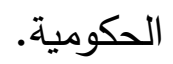

ثانياً: خصائص التجارة الاكترونية(1) 1 - الإعتماد على الوسيط الإلكتروني:

إذ أن التعاملات بين المشاركين في هذه التجارة تتم من خلال وسائل إلكترونية

كأجهزة الكمبيوتر التي تكون موصولة بمختلف الشبكات التي تتمثل أساسا في الشبكة العالميـة المفتوحسة وهـي الانترنـــ Internet أو الثــبكات الخاصــة المغلقـة و هـي الانترانت Intranet أو الاكسترانتExtranet ، وقد يكون هذا الاعتماد كليا بدءا من عرض المنتـوج إلى غايـة تسليمه و ذلك بالنسـبة البعض السـلع والخـمات كبـرامج الكمبيوتر ، التسجيلات الموسيقية، الأفلام، الكتب، التقارير، الأبحاث، الاستشارات...، أو جزئيا بالنسبة لباقي المنتجات المادية أو الخدمات التي تستدعي التعامل المباشر . r

فهي تجارة كونية لا تعرف الحدود بين الدول أو القارات، فيمكن لأي شخص طبيعي أو معنوي أن يتعامل بها بغض النظر عن جنسيته أو مكان تواجده، و هذا الأمـر يطرح عدة إشكالات خاصـة من الناحية القانونية، كالقانون الـلازم تطبيقه في حالة النزاع بين المتعاملين. ب- تطوير منشآت الأعمال و تحسين أدائها: 
لا شك أن التطور السريع في مجال التجارة الإلكترونية دفع منشآت الأعمال إلى التكيف بسرعة حتى تستطيع تلبيـة احتياجات العميل، و بالتالي يكون التجارة الإكترونية أثر كبير على خطط معظم منشآت الأعمال لتصبح هذه التكنولوجيا مألوفة لاى هذه المنشآت على نحو سريع من حيث تعلم و فهم تلك الوسائل التكنولوجية فهما كاملا إلى جانب فهم قوتها لإعادة تثكيل علاقاتها مع العملاء، وبصورة مماثلة تحتاج وسائل التكنولوجيا الجديدة اتجاهات تنظيمية جديدة، فعلى سبيل المثال، ربما يجب تغيير الهيكل التنظيمي الذي يتعامل مع التجارة الإلكترونية عن ذلك الذي يستخدم في المنشأة في الظروف العادية، و ذلك من أجل أن يكون الهيكل التنظيمي أكثر مرونة و

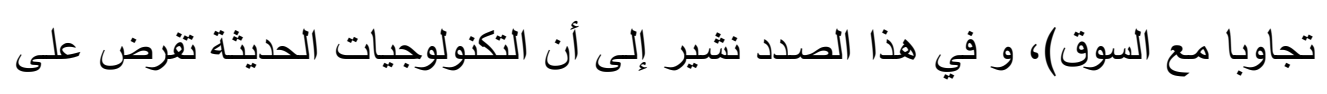
المنشأت تبني الهيكل التنظيمي الثبكي بدل الهرمي.

\section{ثالثاً: أهمية التجارة الإكترونية:(·)}

تمكنت التجارة الإكترونية من إحداث نقلة نوعية هائلة على التجارة، وتمكنت

من تغيير ثقافة الثراء حول العالم، والسلوكيات الثرائية، كما تتميز بأنها ذات منفعة تعود على كل من الأفراد والثركات والمجتـع على حٍٍ سواء، ونشير فيما يلي إلى بعض من هذه الفوائد على النحو التالي: 1أهمية التجارة الإكترونية للشركات: 
مكنت الشركات التجارية من الوصول إلى شريحة أوسع من العملاء المحتملين بسهولة وخلال وقت أقصر من الطرق التجارة التقليدية، مما كان لـه تأثير إيجابي ملحوظ في تحقيق معدلات أعلى من المبيعات، وبالتالي زيادة الأرباح • توفر التجارة الإكترونية مجال أكبر لعرض المنتجات في كل من الأسواق الدحلية والعالمية والدولية أمام شركات الأعمال والثركات التجارية . ساعدت في تهيئة وتطوير المنتجات وفقاً لرغبات المستهلكين؛ وتعتبر من أهم الفوائد التي تعود على الشركات التجارية. مكنت التجار والثركات التجارية على تطوير وتحديث منتجاتهم؛ مما يعني نسب مبيعـات أكبر، حيث يمكن عبر التجـارة الإلكترونيـة معرفـة صدى تجـارب المستهلكين عبر خاصية التقييم التي باتت متوفرة بغالبية المنصات الإلكترونية . تلعب دوراً كبيراً في التقليل من المدة الزمنيـة المستغرقة لإنجاز المعاملات الثرائية، إذ قللت من الفترة الفاصلة بين عملية الثراء وسداد القيمة . r-أهمية التجارة الإكترونية للأفراد: إتاحة الفرصة أمام كبار التجار وأصحاب رؤوس الأموال الصغيرة من إنجاز المعاملات التجارية الخاصة بهم بسهولة وسرعة . ساهمت في التخلص من قيود الزمان والمكان . 
مكنت نسبة كبيـرة مـن الأشـخاص بمختلف دول العـالم مـن الشـروع بإقامـة مشاريعهم التجاريـة من منازلهم، ودون الحاجة للخروج من المنزل؛ الأمر الذي ساعد بشكل كبير ربات البيوت الراغبين بتحقيق دخل إضافي • ساهدت في ظهور مواقع تعليمية إلكترونيـة من أجل تبادل الآراء والخبرات

والمعرفة بين الأشخاص حول العالم، ومن ضمنها كل ما يتعلق بالتجارة الإلكترونية . ب-أهمية التجارة الإكترونية للمجتمع: عملت التجـارة الإكترونيـة على تسهيل ظهيور أنمـاط متعددة مـن الحيـاة الإجتماعية، وخصوصاً بعد أن وفرت فرص عمل أكثر للأفراد من خلال العمل من منـازلهم، الأمـر الذي لعب دوراً كبيراً في التخفيف مـن الأزمـات المروريـة، ومعدل

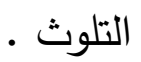
ساعدت فئات المجتمع محدودة الدخل من العثور على متطلباتهم من المنتجات والسلع بأسعار تتلائم مع قدراتهم المادية، وذلك عبر توفير مواقع شراء كثيرة يمكن المقارنة بين أسعار المنتجات فيها، واختيار الأرخص. رابعاً: نتائج البحث ومناقشتها: 1- الأهميه النسبيه لعداد السكان ومستخذمي الانترنت لقاره افريقيا مقارنـه بالعالم

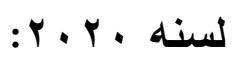
حيث يتضح من خلال الجدول رقم (1)، والثكل البياني رقم (1) أن: هناك تفاوت واضح في انتثار الانترنت عبر العالم، فنجد أن قاره أفريقيا تحتل المرتبه الثانيه 


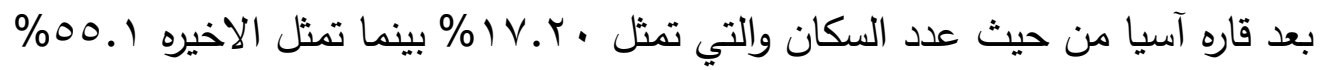
وذلك من اجمالي قارات العالم، كما يتضـح ايضـاً أن قاره افريقيا تحتل المرتبه الثالثه من حيث عدد المستعملين او المستخدمين للانترنت بعد قارتي آسيا وأوروبـا، بينمـا تحتـل القـاره الافريقيـه المرتبـه الآخيـره مـن حيـث النسـبه المئويـه لعـد المسـخدمين للانترنت مقارنـه باجمالي عدد سكان نفس القاره حيث تمثل ادني نسبه والتي تبلغ

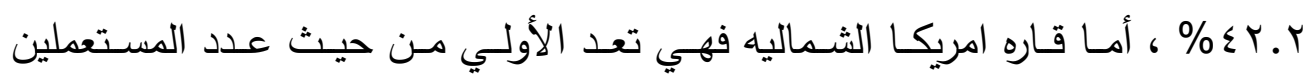
للانترنت والتي تمثل ؟ . 9\% ، ويتوسط القـارتي الخمس قارات العـالم الاخر وهـ أوروبـا، أمريكا اللاتينيه، الثرق الأوسط، استراليا، آسيا ، وتقدر النسبه المئويـه لعدد

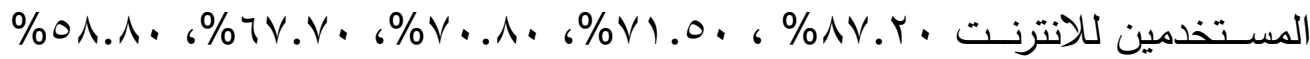
علي الترتيب.

جدول رقم (1): يوضـح الأهميـه النسبيه لعدد السكان ومستخدمي الانترنت لقـاره

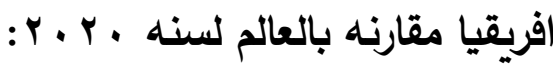

\begin{tabular}{|c|c|c|c|c|}
\hline \% بلعدد مستخدمي & عدد مستخدمي & السكان للعالم عدد & عدد السكان & مناطق العالم \\
\hline 42.2 & 566138772 & 17.20 & 1340598447 & افريقيا \\
\hline 58.80 & 2525033874 & 55.1 & 4294516659 & آسيا \\
\hline 87.20 & 727848547 & 10.70 & 834995197 & أوروبا \\
\hline 71.50 & 467817332 & 8.40 & 654287232 & أمريكا \\
\hline
\end{tabular}




\begin{tabular}{|c|c|c|c|c|}
\hline 70.80 & 184856813 & 3.30 & 260991690 & الأوسط الشرق \\
\hline 90.3 & 332908868 & 4.70 & 368869647 & أمريكا \\
\hline $67.70 \%$ & 28917600 & 0.5 & 42690838 & أستراليا \\
\hline $62.00 \%$ & 4833521806 & $100.00 \%$ & 7796949710 & جمالي العالم \\
\hline
\end{tabular}

www.internetworldstats.com , United Nations Population Division, Website Surfing Guide.

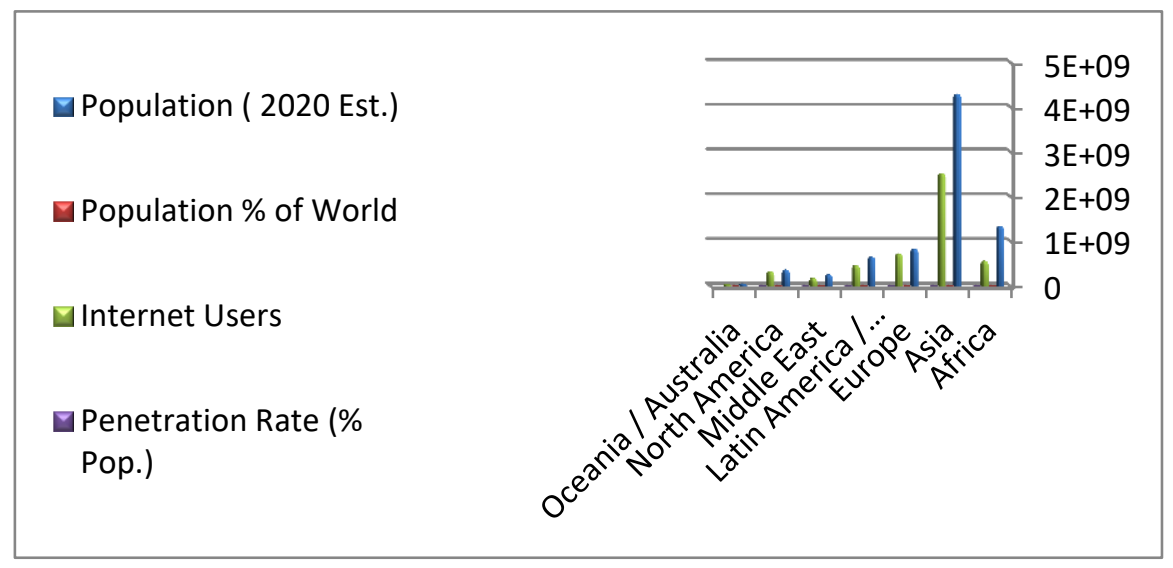

مصدر البيانات: نتائج الجدول رقم (1).

الثكل رقم (1): الأهميه النسبيه لعدد السكان ومستخدمي الانترنت لقاره افريقيا

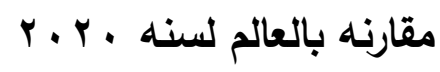

الأمـر الذي يجعل مـن الضـروره التعـرف علي مـدي انتشـار في دول القـاره الافريقيه بوجه خاص، والاستفاده من مزايا الشبكه العنبكوتيه في التجارة الالكترونية، حيث انها تساهم في الناتج المحلي الاجمالي والذي يظهر بشكل واضـح علي معدل النمو الاقتصادي.

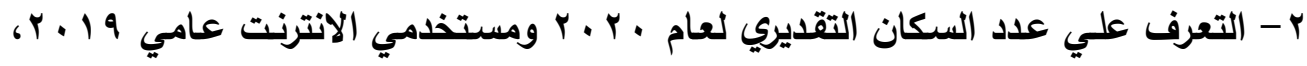
$: r \cdot r$. 


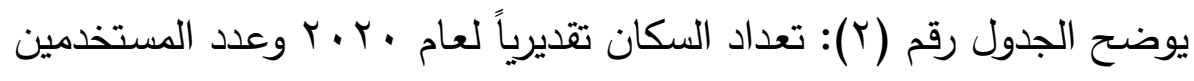

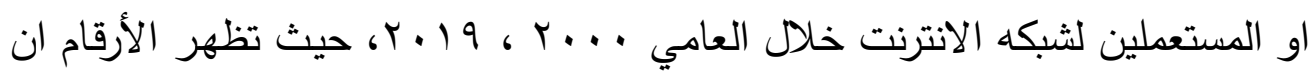
دولة سانت هيلانة تمثل اقل عدد سكان في دول القاره الافريقية حيت تمثل حوالي

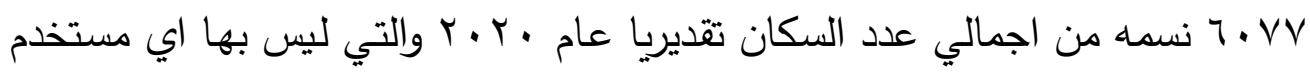
لثبكه الانترنت علي الاطـلاق عـام ....ب، ولكن مرورا بالفتره الزمينه حتي عـام

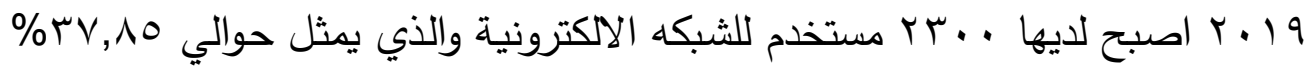
من اجمالي عدد سكانها، ففي عام ... ب كانت هذه الدول مايوت، سانت هلانة، جنوب السودان ، الصحراء الغربيه لا يوجد بها اي مستخدم لثبكه الانترنت، وكانت دولة الصومال أقل عددا لمستخدمي للانترنت وكان بها .. م مستخدم فقط لثبكه

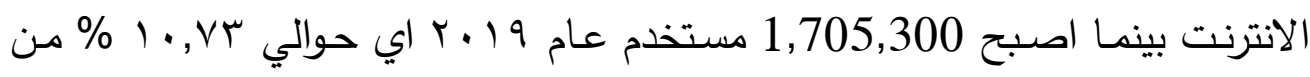
اجمالي عدد السكان وهو 15,893,222 نسمه، امـا جنوب أفريقيا فكان يمثل اكبر عدد لمستخدمي الانترت في ذات العام .... لدول القاره وهو 2,400,000 شخص وهو علي العكس تمامـا لدولة نيجيريا والتي يبلغ سكانها تقديريا لعام · · · حوالي 206,139,589 نسمه والذي يمثل اكبر عدد للسكان في دول القارة الافريقيه أي

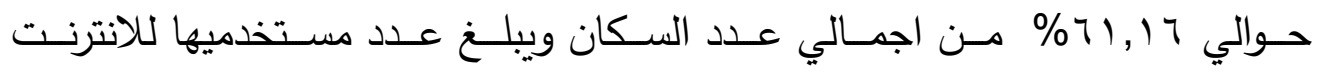
126,078,999 شخص بها ، وعلي الرغم من انها الدوله الوحيده الاعلي سكانا في دول القاره الافريقيه الا ان دولهـ كينيا والتي بها 53,771,296 شخص من اجمالي 


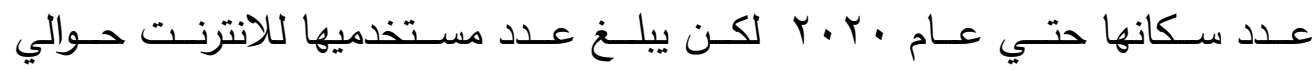

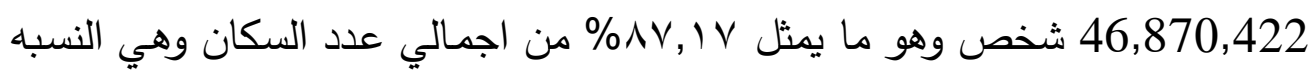
الأعلي لمستغلي شبكه الانترنت وهو مايعني أن الغالبيه العظمي من الافراد القانطين بدولة كينيا يستخدمون الانترنت امـا الصـحراء الغربيه فكانت اقل نسبه لسستخدي الانترنت وهي 4.69\% وذلك من اجمالي سكانها التقديري لعام •r ·r والذي يبلغ

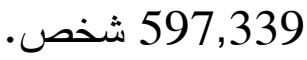

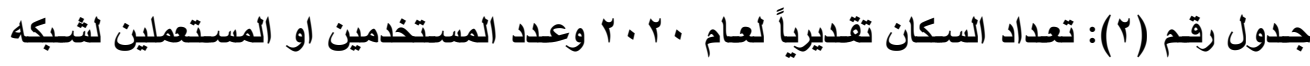

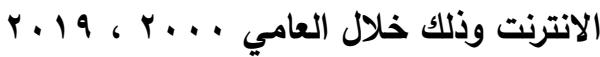

\begin{tabular}{|c|c|c|c|c|}
\hline " من اجعدا المستذمين عد & 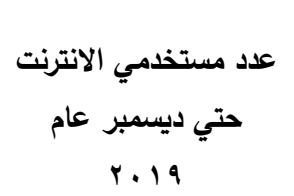 & 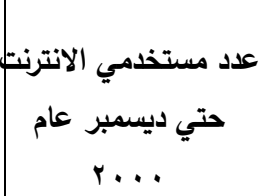 & 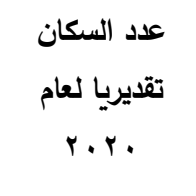 & دول القاره \\
\hline 57.99 & $25,428,159$ & 50,000 & $43,851,044$ & الجزائر \\
\hline 21.54 & $7,078,067$ & 30,000 & $32,866,272$ & أنغولا ل أنولا \\
\hline 31.36 & $3,801,758$ & 15,000 & $12,123,200$ & بنين \\
\hline 47.46 & $1,116,079$ & 15,000 & $2,351,627$ & بوتسوانا \\
\hline 17.72 & $3,704,265$ & 10,000 & $20,903,273$ & بوركينا فاسو \\
\hline 9.71 & $1,154,568$ & 3,000 & $11,890,784$ & بوروندي \\
\hline 63.33 & 352,120 & 8,000 & 555,987 & كابو فيردي \\
\hline 23.09 & $6,128,422$ & 20,000 & $26,545,863$ & الكاميرون \\
\hline 13.57 & 655,466 & 1,500 & $4,829,767$ & جمهورية أفريقيا \\
\hline 6.26 & $1,027,932$ & 1,000 & $16,425,864$ & تثاد \\
\hline 20.52 & 178,500 & 1,500 & 869,901 & جز القُر \\
\hline 13.28 & 732,800 & 500 & $5,518,087$ & الكونغو \\
\hline 8.35 & $7,475,917$ & 500 & $89,561,403$ & جمهورية الكونغو \\
\hline
\end{tabular}




\begin{tabular}{|c|c|c|c|c|}
\hline 45.32 & $11,953,653$ & 40,000 & $26,378,274$ & كوت ديفوار \\
\hline 55.55 & 548,832 & 1,400 & 988,000 & جيبوتي \\
\hline 48.11 & $49,231,493$ & 450,000 & $\begin{array}{c}102,334,40 \\
4\end{array}$ & مصر \\
\hline 25.44 & 356,891 & 500 & $1,402,985$ & غينيا الإستوائية \\
\hline 8.27 & 293,343 & 5,000 & $3,546,421$ & إريتريا \\
\hline 57.34 & 665,245 & 10,000 & $1,160,164$ & إيسواتيني \\
\hline 17.84 & $20,507,255$ & 10,000 & $\begin{array}{c}114,963,58 \\
8 \\
\end{array}$ & أثيوبيا \\
\hline 58.75 & $1,307,641$ & 15,000 & $2,225,734$ & الجابون \\
\hline 18.29 & 442,050 & 4,000 & $2,416,668$ & غامبيا \\
\hline 37.78 & $11,737,818$ & 30,000 & $31,072,940$ & غانا \\
\hline 18.36 & $2,411,672$ & 8,000 & $13,132,795$ & غينيا \\
\hline 12.70 & 250,000 & 1,500 & $1,968,001$ & غينيا بيساو \\
\hline 87.17 & $46,870,422$ & 200,000 & $53,771,296$ & كينيا \\
\hline 31.88 & 682,990 & 4,000 & $2,142,249$ & ليسوتو \\
\hline 12.35 & 624,610 & 500 & $5,057,681$ & ليبيريا \\
\hline
\end{tabular}

www.internetworldstats.com , United Nations Population Division , Website Surfing Guide.

*تم الحصول عليها بخارج قسمه عدد المستخدمين مقسوما علي اجمالي عدد السكان مضروبا في . . 
تابع جدول رقم (r): تعداد السكان تقديرياً لعام • r • و وعدد المستخدمين او المستعملين لشبكه

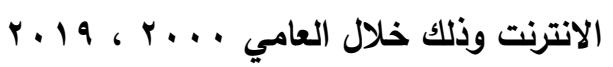

\begin{tabular}{|c|c|c|c|c|}
\hline 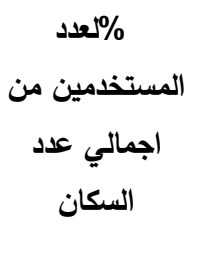 & عد عـي ديسمبر عام الانترنت & $\begin{array}{c}\text { عدد مستخدمي الانترنت ديسمبر عام } 2 . . . \\
\text { ع... }\end{array}$ & عدام السكان تقديريا & دول القاره \\
\hline 74.22 & $5,100,000$ & 10,000 & $6,871,292$ & ليبيا \\
\hline 9.54 & $2,643,025$ & 30,000 & $27,691,018$ & مدغشقر \\
\hline 14.20 & $2,717,243$ & 15,000 & $19,129,952$ & ملاوي \\
\hline 61.63 & $12,480,176$ & 18,800 & $20,250,833$ & مالي \\
\hline 20.85 & 969,519 & 5,000 & $4,649,658$ & موريتانيا \\
\hline 66.99 & 852,000 & 87,000 & $1,271,768$ & موريشيوس \\
\hline 39.57 & 107,940 & لا يوجد & 272,815 & مايوت (فرنسا) \\
\hline 64.32 & $23,739,581$ & 100,000 & $36,910,560$ & المغرب \\
\hline 20.87 & $6,523,613$ & 30,000 & $31,255,435$ & موزمبيق \\
\hline 53.03 & $1,347,418$ & 30,000 & $2,540,905$ & ناميبيا \\
\hline 11.49 & $2,781,266$ & 5,000 & $24,206,644$ & النيجر \\
\hline 61.16 & $126,078,999$ & 200,000 & $206,139,589$ & نيجيريا \\
\hline 61.77 & 553,000 & 130,000 & 895,312 & ريونيون \\
\hline 46.18 & $5,981,638$ & 5,000 & $12,952,218$ & رواندا \\
\hline 37.85 & 2,300 & لا يوجد & 6,077 & سانت هيلانة \\
\hline 29.14 & 63,864 & 6,500 & 219,159 & ساو تومي \\
\hline 58.23 & $9,749,527$ & 40,000 & $16,743,927$ & السنغال \\
\hline 72.50 & 71,300 & 6,000 & 98,347 & سيشيل \\
\hline 13.08 & $1,043,725$ & 5,000 & $7,976,983$ & سيرا ليون \\
\hline 10.73 & $1,705,300$ & 200 & $15,893,222$ & الصومال \\
\hline 54.99 & $32,615,165$ & $2,400,000$ & $59,308,690$ & جنوب أفريقيا \\
\hline 7.93 & 887,722 & لا يوجد & $11,193,725$ & جنوب السودان \\
\hline 29.93 & $13,124,100$ & 30,000 & $43,849,260$ & السودان \\
\hline 38.74 & $23,142,960$ & 115,000 & $59,734,218$ & تنزانيا \\
\hline
\end{tabular}




\begin{tabular}{|c|c|c|c|c|}
\hline 12.22 & $1,011,837$ & 100,000 & $8,278,724$ & توجو \\
\hline 66.83 & $7,898,534$ & 100,000 & $11,818,619$ & تونس \\
\hline 40.45 & $18,502,166$ & 40,000 & $45,741,007$ & أوغندا \\
\hline 4.69 & 28,000 & لا يوجد & 597,339 & الغرحراء \\
\hline 53.69 & $9,870,427$ & 20,000 & $18,383,955$ & زامبيا \\
\hline 56.52 & $8,400,000$ & 50,000 & $14,862,924$ & زمبابوي \\
\hline 39.29 & $526,710,313$ & $4,514,400$ & $1,340,598,447$ & إجمالي أفريقيا \\
\hline 62.87 & $4,058,868,405$ & $82.80 \%$ & $6,456,017,263$ & باقي العالم \\
\hline 58.81 & $4,585,578,718$ & $100.00 \%$ & $7,796,615,710$ & إجمالي العالم \\
\hline
\end{tabular}

ومـن هذا المنطلق يتبـين مجمـلاً أن نسبه عدد المستخدمين لشـبكه الانترنـت للقـاره الافريقيـه هـي 0. 11\% فقط مقارنه بقارات العالم، وهي نسبه ضئيله جدا تشير الي عدم الاستفاده الكامله من شبكه الانترنت والحصول علي مزاياها لافراد تلك الدول، ومن ثم وجب التعرف علي العقبات التي تواجه دول هذه القاره لنمو التجارة الاكترونية.

\section{Internet Users in Africa 2020 Q1 - March}

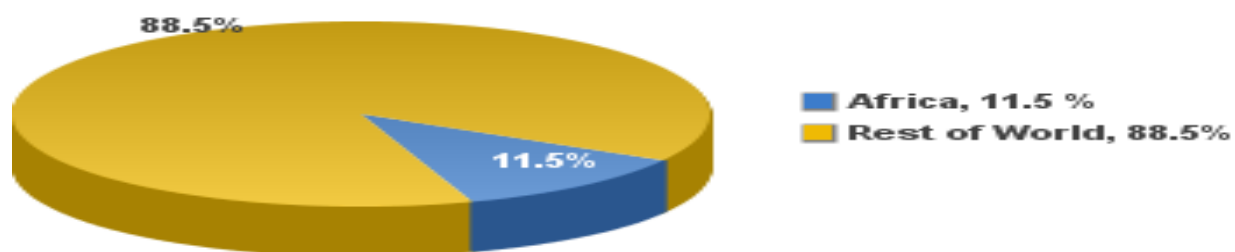

Source: Internet World Stats - mmw.internetworldstats.com

$526,710,313$ estimated Internet users in Africa in March, 2020 , representing $11.5 \%$ of the total world Internet users, estimated at 4,585,578,718. Copyright@2020, Miniwatts Marketing Group

خامساً: العقبات التي تواجه نمو التجارة الإلكترونية في أفريقيا: (؛) هناك العديد من العقبات التي تحول دون نمو التجارة الإلكترونية وتقدمها في أفريقيا: ففي الوقت الذي يصل فيه عدد الشركات الناشئة التي تدخل السوق التجاري الإلكتروني في أفريقيا نحو ع ب شركة تعمل في جميع أنحاء القارة: فإن أقل من • ب من الثركات الأفريقية الناشئة في مجال التجارة الإكترونية تعد شركات مربحة؛ 
لأن باقي تلك الشركات تواجه مشكلات عديدة تحول دون تقدمها وتوسعها في القارة، ومنها ضعف الثقة، والصعوبات اللوجستية، وصعوبات النقل والثحن ... وغيرها، ومن

$$
\text { أهم تلك العقبات ما يلي:- }
$$

• ضـعف تواصل المواطنين مـع شبكات الإنترنت: فوفقا لدراسة أجرتها شركة

Deloitte

اتصال بالإنترنت. هذه التغطية: على الرغم من زيادتها التدريجية الثابتة لتصل

إلى 0ب\% عام 9 ـ ـ ب، إلا أنها لا تزال بحاجة إلى مزيد من التحسين سواء

من حيث توفير شبكات الإنترنت وتحسين مستوى جودتها، والحد من تكاليفها

المرتفعة حتى يمكن جذب أكبر عدد ممكن من المستهلكين الأفارقة.

• ضــف البنيـة التحتيـة والخدمات اللوجستية: مـن أكبر العقبـات التي تواجـه

التجارة الإكترونية في أفريقيا هي ضعف البنية التحتية: حيث الطرق الضعيفة

التي لا تسمح بالنقل السريع للسلع، وبالتالي وصولها إلى العملاء بشكل أسرع

وكفاءة عالية، كذلك عدم وجود أنظمة عناوين وطنية مناسبة في معظم الدول

الأفريقية؛ حيث تعتمد الثركات على العناوين والمعالم الوصفية إلى حد مـا

والتي قدمها العملاء خلال المراحل الأولية من عملية الثراء عبر الإنترنت،

ومعظمها لا يوفر معلومات واضحة عن المكان المحدد للعمـلاء ويؤدي إلى

تأخر في عمليات الثـحن، ويشير تقرير لبنك التنمية الأفريقي عن الآفـاق 
الاقتصـادية الأفريقيـة لعـام 9 ـ ب إلى أن تكاليف التجارة الإلكترونيـة بسبب ضعف أداء أسواق اللوجستيات قد تكون حاجزا أمام التجارة أكبر من التعريفات الجمركية والحواجز غير الجمركية". وإن كانت بعض الدول الأفريقية قد سجلت أداء أفضل من حيث البنية التحتية واللوجستية، ففي تقرير للبنك الدولي عام 1 ا ـ Y: وضـع مؤشر الأداء اللوجستي كلا من جنوب أفريقيا وكينيا ورواندا وكوت ديفوار في صدارة الأربع دول الأفضل أداء في أفريقيا، وبدأت بعض الشركات العاملة في مجال التجارة الإلكترونية تفكر في سبل أسرع لتوصيل السلع وطرق نقل بديلة، ومنها استخدام الدراجات النارية سهلة التنقل والمعروفة باسم بودابوداس Bodabodas التي تم استخدامها في دول مثل كينيا ونيجيريا لتجنب الطرق المزدحمة في المدن الكثيفة من حيث عدد السكان. • إرتفاع التكاليف المرتبطة بالثحن عبر الحدود: مما يزيد من عملية تأخير نقل البضــائع، فضـلا عـن ضــف خـمات البنيـة التحتيـة المتعلقـة بالثـحن مثل اللوجستيات، كذلك خدمات البريد التي يضعف الثقة فيها في أفريقيا، وقد لا تتوافر إلا في عدد محدود من المدن الكبرى في أفريقيا. • ضعف الثقة: يزداد تخوف المواطنين من عمليات البيع والثراء عبر الإنترنت؛ نظرا لتعرضـهم لحالات النصب والخداع والاحتيال الإكتروني، حيث حالات الاختراق عبر البريد الإلكترونـي، أو التحصيل المسبق لرسوم الثـحن وثـن 
السلع دون توصيلها إلى المشترين، ومن هنا لا يشعر العديد من الأشخاص بالأمسان عند وضـع معلومـاتهم الماليـة؛ مثل بطاقات الائتمان على المواقع

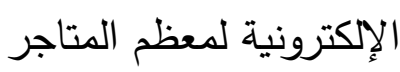
انحصـار طـرق الـفقع في الـدفع النقدي: يفضـل معظم الأفارقـة دفـع ثـنـ مشترياتهم الإلكترونية نقدا عند استلامها: فنحو 9\% من عمليات الثراء عبر الإنترنت تتم بهذه الطريقة، وهي الطريقة التي تناسب معظم الأفارقة، حيث لا يملك سوى ما نسبته ـ 1- 10 \% فقط من السكان حسابا مصرفيا يمكن من خلاله تحويل الأموال عبر الإنترنت بدلا من الدفع النقدي، وهو ما لا يساعد على تقدم ونمو التجارة الإكترونية ـ ولا تزال نسبة كبيرة من السكان الأفارقة بدون حساب مصرفي كما تؤكد تقديرات صندوق النقد الدولي أن ·r\% فقط من السكان لديهم حساب مصرفي وإن كانت هناك بعض الدول الأفريقية مثل جنوب أفريقيا لديها أعلى معدل انتثـار للإنترنت، وأعلى نسبة من السكان الذين يمتلكون حسابات مصرفية ومالية مقارنة بدول أفريقيا جنوب الصحراء، إلا أنها ما زالت تعاني أيا من قلة استخدام بطاقات الائتمان و خدمات نقل الأموال عبر الهواتف المحمولة. • الاسواق المجزأة: حيث يوجد في أفريقيا عدد كبير جدا من الدول ذات التتوع الكبير - ثقافيـا واقتصـاديا وسياسيا. وهذا يخلق 
العديد من الحواجز سواء في اللغات أو الثقافة والعادات الاستهلاكية، وطرق المدفوعات عبر الحدود... وغيرها من الحواجز، مما يجعل من الصعب على مواقع التجارة الإلكترونية التوسع بسهولة في أفريقيا والتتمع بوفورات الحجم الكبير • وللتخفيف من هذا التحدي يجب على الدول الأفريقية الاستمرار في خطوات التكامل القاري الاقتصـادي من أجل مواءمـة ظروف العمل لمعظم

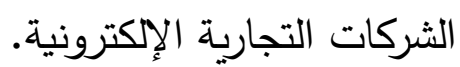
• ارتفاع معدلات الأمية: يعني أن العديد من الأفراد لا يمكنهم المشاركة مباشرة على منصات التجارة الإكترونية نظرا لعدم إجادتهم القراءة أو الكتابة؛ وبالتالي أصعب ما يكون عليهم التواصل عبر الإنترنت، وهو ما يجعل أفريقيا بحاجة إلى مواصلة الاستثمار في التعليم بقوة لزيادة عدد الأثخاص الذين يمكنهم القراءة والكتابة والحد من معدلات الأمية التي تصب في غير صالح التتمية

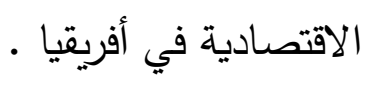




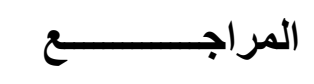

اولاً: دوريات عربيه:

1- بن وارث حكيمـه، دور وأهميـه التجـارة الاككترونيـة في اقتصـاد المعرفهـ مـع الاشـاره الي العـالم العربي، ، مذكرة مقدمة ضمن متطلبات نيل شهادة الماجستير في العلوم الاقتصادية، معهد العلوم الاقتصادية وعلوم التسيير دائرة مابعد التدرج، جامعة أم البواقي، الجزائر، ؟ .. ؟. ץ- رأفت رضوان ، عالم التجارة الإكترونية ، المنظمة العربية للتتمية الإدارية ، القاهرة 1999 ، ص

ז- مراد رايس (دكتور)، عبدالهادي مسعودي (دكتور)، عوائق اعتماد التجارة الاككترونية في الوطن العربي والاسـلامي، الملتقـي العلدي الدولي الخـامس حول الاقتصـاد الافتراضسي وانعكاسـاته علي الاقتصاديات الدولية، جامعة عمار ثليجي، الأغواط، الجزائر. ع - مركز الفاروس للدارسات والاستثارات الاستراتيجيه، مركز بحثي مختص بالثئون الافريقية، قسم دراسات العلاقات الدولية، القاهرة، جمهورية مصر العربية، ·. ·.

ثانياً: دوريات أجنبية: 5- Nicolas Goldstein, The Future of E-commerce In Africa: A Mere Illusion? , 2019

ثالثاً: مواقع هيئات رسميه دوليه: 6-بيانات الأمم المتحده والاتحاد الدولي للاتصالات 7-احصائيات البنك الدولي

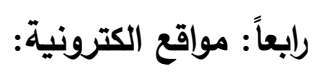


https://pharostudies.com/?p=5142-8

https://www.mcit.gov.sa/ar/media-center/news/95557-9

https://read.opensooq.com - 1 .

www.internetworldstats.com - 11 